\title{
Amygdala Activation for Eye Contact Despite Complete Cortical Blindness
}

\author{
Nicolas Burra, ${ }^{2,3}$ Alexis Hervais-Adelman, ${ }^{3,4}$ Dirk Kerzel, ${ }^{2,3}$ Marco Tamietto, ${ }^{5,7}$ Beatrice de Gelder, ${ }^{5,6}$ \\ and Alan J. Pegna ${ }^{1,2,3}$ \\ ${ }^{1}$ Laboratory of Experimental, Neuropsychology Unit/Neurology Clinic, Department of Clinical Neuroscience and Dermatology, Geneva University Hospital, \\ CH-1211 Geneva, Switzerland, ${ }^{2}$ Faculty of Psychology and Educational Science, University of Geneva, CH-1211 Geneva, Switzerland, ${ }^{3}$ Neuroscience Center, \\ University of Geneva, CH- 1211 Geneva, Switzerland, ${ }^{4}$ Brain and Language Laboratory, University of Geneva Medical School, University of Geneva, \\ CH- 1211 Geneva, Switzerland, ${ }^{5}$ Cognitive and Affective Neurosciences Laboratory, Tilburg University, 5000 AB Tilburg, The Netherlands, ${ }^{6}$ Athinoula A. \\ Martinos Center for Biomedical Imaging, Massachusetts General Hospital-Harvard Medical School, Charlestown, Massachusetts 02129, and \\ ${ }^{7}$ Department of Psychology, University of Torino, 10126 Torino, Italy
}

Cortical blindness refers to the loss of vision that occurs after destruction of the primary visual cortex. Although there is no sensory cortex and hence no conscious vision, some cortically blind patients show amygdala activation in response to facial or bodily expressions of emotion. Here we investigated whether direction of gaze could also be processed in the absence of any functional visual cortex. A well-known patient with bilateral destruction of his visual cortex and subsequent cortical blindness was investigated in an fMRI paradigm during which blocks of faces were presented either with their gaze directed toward or away from the viewer. Increased right amygdala activation was found in response to directed compared with averted gaze. Activity in this region was further found to be functionally connected to a larger network associated with face and gaze processing. The present study demonstrates that, in human subjects, the amygdala response to eye contact does not require an intact primary visual cortex.

\section{Introduction}

It has long been known that a loss of occipital striate cortex leads to blindness of cortical origin (e.g., Holmes, 1918). Although such lesions obviously deprive a person of any conscious visual sensation, evidence has accumulated showing that a substantial amount of visual processing still remains possible without awareness. This residual capacity has been termed "blindsight" (Weiskrantz et al., 1974) and has been observed in a number of different tasks, such as motion detection, target localization, or pointing (Weiskrantz, 1986).

If evidence of blindsight was found for what could perhaps be described as basic visual characteristics, subsequent observations showed that more complex, emotional stimuli also produced nonconscious processing. Indeed, de Gelder et al. (1999) discovered that emotional faces were processed even when they were presented to a patient's blind field and thus could not induce any conscious percept, thereby demonstrating the existence of an "affective" blindsight. Subsequently, another patient (T.N.), who is

\footnotetext{
Received Aug. 21, 2012; revised May 15, 2013; accepted May 21, 2013.

Author contributions: N.B., D.K., B.d.G., and A.J.P. designed research; N.B., A.H.-A., M.T., B.d.G., and A.J.P. performed research; N.B., A.H.-A., M.T., B.d.G., and A.J.P. analyzed data; N.B., A.H.-A., D.K., M.T., B.d.G., and A.J.P. wrote the paper.

This work was supported by the Swiss National Science Foundation for Scientific Research Grants 320030 144187 and PDFMP1_129459.

The authors declare no competing financial interests.

Correspondence should be addressed to Dr. Alan J. Pegna, Laboratory of Experimental Neuropsychology, Neuropsychology Unit/Neurology Clinic, Geneva University Hospital, 4 Gabrielle-Perret-Gentil, CH-1211 Geneva 4, Switzerland. E-mail: alan.pegna@hcuge.ch.

DOI:10.1523/JNEUROSCI.3994-12.2013

Copyright $\odot 2013$ the authors $\quad 0270-6474 / 13 / 3310483-07 \$ 15.00 / 0$
}

the subject of the current report, also proved to be able to guess, with a success rate significantly above chance, whether a positive or negative facial expression was presented to him (Pegna et al., 2005). fMRI revealed that this was possible through activity of his right amygdala, even though V1 was completely destroyed in this person.

The fact that emotional stimuli can be processed without V1 and without awareness is in line with the assumption that elements important for our survival, in particular threatening stimuli, may be processed through a coarse and rapid subcortical pathway (LeDoux, 1998) to the amygdala. However, it seems unlikely that this pathway would only process emotional expressions because other facial cues are equally behaviorally relevant and could be necessary for the rapid and automatic appraisal of a situation.

One stimulus of high significance in humans is the direction of gaze. Indeed, direction of gaze provides important indications regarding another person's intentions and thus yields essential information about avoidance or approach behavior. We hypothesized that, as for emotional faces, gaze may be processed in the absence of awareness, bypassing primary sensory areas and accessing relevant brain structures through alternate channels. Furthermore, previous studies have shown that emotional facial expressions activate the amygdala when high spatial frequencies are filtered out, although this does not hold for stimuli removed of their low spatial frequencies (Vuilleumier et al., 2003). This was taken as evidence that the amygdala processes mainly the low spatial frequency of the stimuli.

Consequently, using an fMRI block-design paradigm, we investigated the cerebral response to gaze in Patient T.N., who pres- 
ents complete cortical blindness resulting from bilateral loss of his visual cortex. Photographs of faces, with high, low, or no spatial frequency filtering, were presented with their gaze oriented toward or away from him with the aim of determining whether structures, such as the amygdala, would respond to eye contact despite the patient's inability to report the presence of any visual stimulation. In addition, a group of healthy controls matched for education, sex, and age also performed the task for comparison purposes.

\section{Materials and Methods \\ Case reports}

Patient T.N. is a 57-year-old man trained and still working as a physician. He suffered two consecutive strokes at the age of 52, the first in the left parieto-temporo-occipital cerebral area, which initially produced right hemianopia, hemiplegia, and transcortical sensory aphasia, which receded rapidly except for the hemianopia. A second hemorrhage occurred in the right occipital lobe producing a loss of the remaining (left) visual field.

Structural MRI shows that the lesion in the left hemisphere includes most of the occipital lobe, with minimal sparing of the medial ventral part of the inferior occipital gyrus and anterior part of the lingual gyrus. The lesion extends anteriorly to the middle part of the fusiform gyrus leaving the parahippocampal gyrus grossly intact. Laterally, the lesion extends to the medial inferior temporal gyrus. Dorsally, the hemorrhage reaches the superior parietal lobule and spares the ventral part of the precuneus. The right hemisphere lesion is smaller and includes most of the occipital lobe, with limited sparing of the medial part of the posterior lingual gyrus and medial part of precuneus. The anterior border stretches to the middle part of the fusiform gyrus and included the posterior inferior temporal gyrus but spares the parahippocampal gyrus. No anatomic input to striate areas could be detected in either left or right hemisphere using DTI.

Stimuli. Six different identities ( 3 male, 3 female) were created using FaceGen Modeler 3.4, a software that has been already used in previously published experiments on gaze perception (see, e.g., Ethofer et al., 2011). Using ImageJ and a procedure suggested by other authors (Delplanque et al., 2007), these avatars were desaturated and equalized for their facial luminance (Fig. 1). Presented on a gray background, each picture, $512 \times$ 512 pixels, was then filtered using the parameters usedin a previous paradigm (Vuilleumier et al., 2003). This yielded three filtering conditions: normal (unfiltered) pictures, low-pass filtered pictures ( 6 cycles/image), and high-pass filtered pictures ( 32 cycles/ image). Using FaceGen settings for the gaze deviation ( $50 \%$ of the maximal deviation), we deviated the averted gaze positions in $50 \%$ of the presentations to the left and $50 \%$ to the right. For the behavioral experiment, a pure tone at $440 \mathrm{~Hz}$ was generated using Audacity 1.2.6.

Behavioral procedure. The stimuli were presented on a computer screen situated at a viewing distance of $85 \mathrm{~cm}$ (outside the MRI scanner). Half of the stimuli showed a face with an averted gaze (AG), and half depicted a face with its gaze directed at the viewer (DG). Stimuli were presented in a random order, and Patient T.N. was asked to press one of two keys to respond whether the gaze was directed at him or not. Responses were cued by a brief tone $(440 \mathrm{~Hz}, 50 \mathrm{~ms})$ indicating the beginning of stimulus presentation. Patient T.N. was asked to respond after the onset of the auditory cue. The next trial was initiated $1100 \mathrm{~ms}$ after the response. Because of the absence of any effect shown by the patient in guessing, the experiment was run twice more, during a brief testing session 2 years later. In the second of these two later tests, we emphasized the importance of giving a rapid and intuitive response and insisted that he
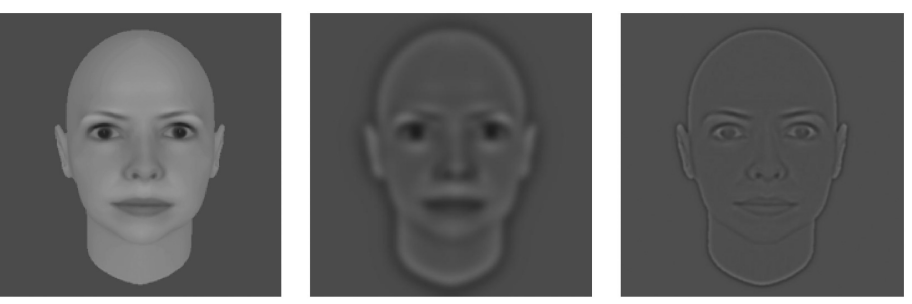

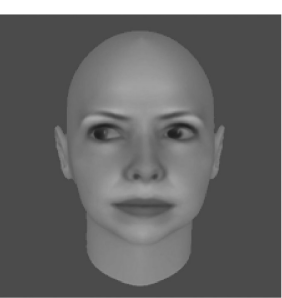

Full

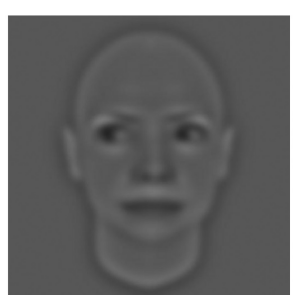

High

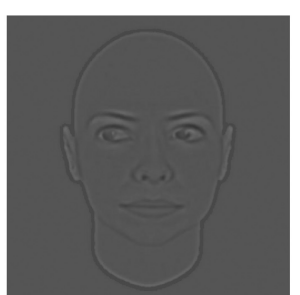

Low

\section{Images spatial frequencies}

Figure 1. Example of stimuli used in this study.

not make any effort but respond with spontaneity to his "gut feeling," instruction that proved fruitful.

fMRI procedure. Patient T.N., lying in the MRI scanner, was requested to simply keep his eyes open during the experimental presentation. Stimuli were projected onto a screen situated at the end of the bore closest to the patient's head. A mirror placed on the head coil reflected the images from the screen directly toward the center of the patient's orbit. After verifying the position of the display mirror (before inserting Patient T.N. into the bore of the scanner), we monitored his gaze direction scrupulously during the recording session with an eye tracker camera, although we were unable to calibrate the eye tracker because of the impossibility for him to fixate calibration points.

We presented 24 blocks of stimuli, each lasting $24 \mathrm{~s}$. Blocks contained 6 tokens of a single condition presented for $2 \mathrm{~s}$ each (direct, averted, direct low spatial frequency, averted low spatial frequency, direct high spatial frequency, averted high spatial frequency) and were presented in a pseudo-random order. Each of the six blocks was separated by a $26 \mathrm{~s}$ rest period with no stimulation. Four blocks of each condition were presented, and each single picture was presented four times per block.

MRI data acquisition. Scanning took place in a 3T Siemens Trio MRI scanner, using a 12-channel head-coil; 340 functional volumes were acquired in one single session, lasting $\sim 12 \mathrm{~min}$ (T2*-weighted EPI, 32 sequential $3 \mathrm{~mm}$ slices, $15 \%$ interslice gap, $2.56 \times 2.56 \mathrm{~mm}$ in-plane resolution, angled away from the eyes to prevent ghost artifacts from aliasing of eye movements; TA, $2 \mathrm{~s}$; TR, $2 \mathrm{~s}$; TE, $30 \mathrm{~ms}$ ). A high-resolution $(0.43 \mathrm{~mm} \times 0.43 \mathrm{~mm} \times 1 \mathrm{~mm}$ voxels $)$ T1-weighted anatomical image was also acquired.

$f M R I$ preprocessing and analysis. Analysis was performed using the SPM 8 software package (http://www.fil.ion.ucl.ac.uk/spm/), with the following steps: (1) realignment, in which EPI volumes were realigned using a two-pass procedure (images were initially realigned to the first volume, and mean image was generated, to which the EPI volumes were then realigned); (2) coregistration of the structural volume to the mean EPI image; and then (3) spatial smoothing using a Gaussian kernel of 8 $\mathrm{mm}$ at full-width half-maximum height.

To be able to compare the patient's neuroanatomy with that of existing atlases, we normalized the T1 anatomical image to the standard MNI template included in SPM8, using the "unified segment" procedure, which is robust in the face of lesions (Ashburner and Friston, 2005; Crinion, 2007). The normalization parameters generated by this process were subsequently applied to the contrast images produced by the functional analysis. Activation coordinates are reported in MNI space, and region identification was determined using the SPM Anatomy Toolbox (Eickhoff et al., 2005). 
a

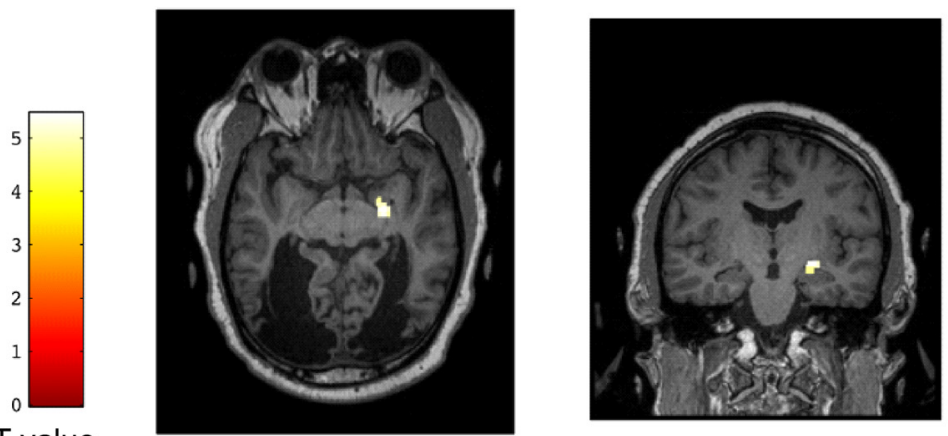

\section{b T-value}

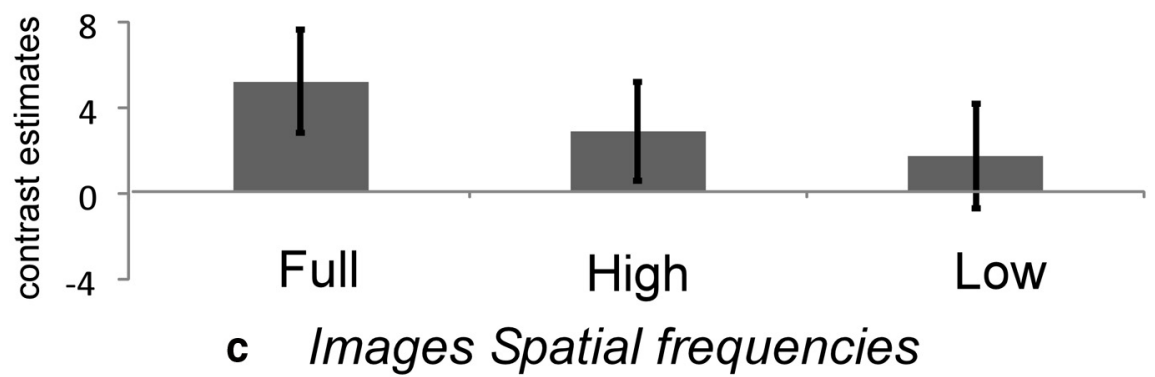

C Images Spatial frequencies

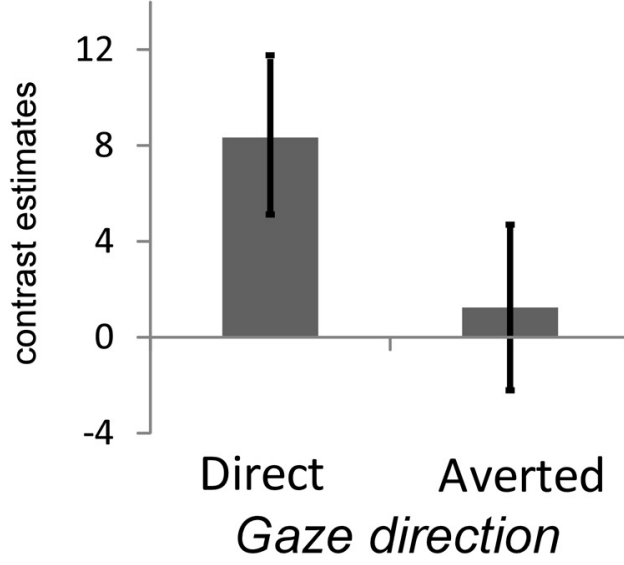

Figure 2. BOLD response in Patient T.N. when presented with photographs of direct gaze (DG) versus averted gaze (AG). $\boldsymbol{a}, A$ significant activation was found in Patient T.N.'s right amygdala when blocks of faces with DGs were contrasted with photographs of faces with AGs. Using a fixed model, voxels showing a significantly greater response $\left(p_{(\mathrm{FWE})}<0.05\right)$ for DG when contrasted with AG were displayed on Patient T.N.'s T1 structural scan. A significantly increased response ( $z$-score $=5.02, p_{(\mathrm{FWE})}$ corrected $=$ 0.004 ) was found in the right amygdala (MNI coordinates, $24 ;-12 ;-10$; volume, $67 \mathrm{~mm}^{3}$ ), consistent with previous findings regarding gaze processing in normally sighted individuals (Kawashima et al., 1999) and affective blindsight in this patient (Pegna et al., 2005). $\boldsymbol{b}, \beta$ values representing activation in the right amygdala (MNI coordinates, $24 ;-12 ;-10)$ for nonfiltered (normal), high-pass filtered and low-pass filtered images, showing no differences in-between conditions. The values illustrated here were obtained from the most significant voxel in the DG versus AG contrast and were not used for statistical computation. c, Contrast estimates were significant for DG versus AG (bars indicate $90 \%$ confidence intervals).

Nonetheless, it is important to note that all figures, $\beta$ values, and functional connectivity analyses that we report were performed on the data in Patient T.N.'s native space.

We used a general linear model in which every scan was coded for condition. The BOLD response to each stimulus was modeled using the canonical hemodynamic response function, along with its temporal and dispersion derivatives. Six movement parameters $(x, y$, and $z$ translations and $x, y$, and $z$ rotations) derived from the realignment step described above were included in the model. A high-pass filter (cutoff $128 \mathrm{~s}$ ) and AR1 correction for serial autocorrelation were applied.

As there was no significant main effect of spatial frequency $\left(p_{(\mathrm{FWE})}>\right.$ 0.05 ) on brain activity, we collapsed the data across all spatial filtering conditions, leaving a single factor: gaze direction.

PPI of the right amygdala. A psychophysiological interaction (PPI) (Friston et al., 1997) analysis was performed to determine regions showing functional connectivity with the right amygdala that was modulated by gaze direction. A PPI is defined as a stimulus-dependent or context-dependent change in the influence of one brain region on another. A spherical ROI with radius $4 \mathrm{~mm}$ was created, centered on the voxel showing the maximum contrast in response to direct versus averted gaze and the eigenvariate of the entire BOLD signal time course corrected for effects of interest was extracted.

\section{Control group}

Ten right-handed, age-matched, male participants ( $57 \pm 4$ years of age) served as controls for Patient T.N. None reported any neurological or psychiatric disorders. Participants were naive as to the purpose of the experiment. The local ethics committee had approved the study, and informed consent was obtained from participants before the experiment.

fMRI data acquisition, preprocessing, and analysis. Data acquisition and procedures for controls were identical to those for Patient T.N. fMRI data were preprocessed as for Patient T.N., with an additional spatial normalization step performed before analysis. Individual participants' datasets were then analyzed using a fixed effects model exactly as for Patient T.N. Contrast estimates for the effects of gaze direction and frequency were calculated. A one-sample $t$ test was then performed on the contrast images obtained from all the subjects for each contrast of interest, treating subjects as a random factor.

Results for the control group are presented at an uncorrected level of $p<0.001$, uncorrected for multiple comparisons, and a cluster extent threshold of 10 voxels.

\section{Results \\ Patient T.N. \\ Behavior}

Patient T.N. was unaware of any of the stimuli presented to him and, in a behavioral task performed outside the scanner, failed to respond above chance when asked to guess whether the gaze were averted or directed at him $(49 \%$ correct responses for gaze contact; $p=$ not significant on a binomial distribution). As noted above, this was the result of the patient's difficulty accepting to guess. Subsequently, a second testing session yielded a similar result ( $48 \%$ correct responses, $p=$ not significant). However in the final session, after having insisted on the necessity of relying on subjective impressions, Patient T.N.'s hit rate rose slightly to 57\% which, although not highly significant, appears less random than during the previous sessions ( $p=0.063$ on a cumulative binomial distribution).

\section{$f M R I$}

fMRI results revealed that the right lateral amygdala produced a larger response for DG than $\mathrm{AG}\left(p_{(\mathrm{FWE})}<0.05\right.$; Fig. 2$)$, whereas no effect was found on spatial filtering. To more closely examine the response of the amygdala as a function of spatial filtering, we performed an additional ROI analysis focused on the amygdalae (discussed below). 


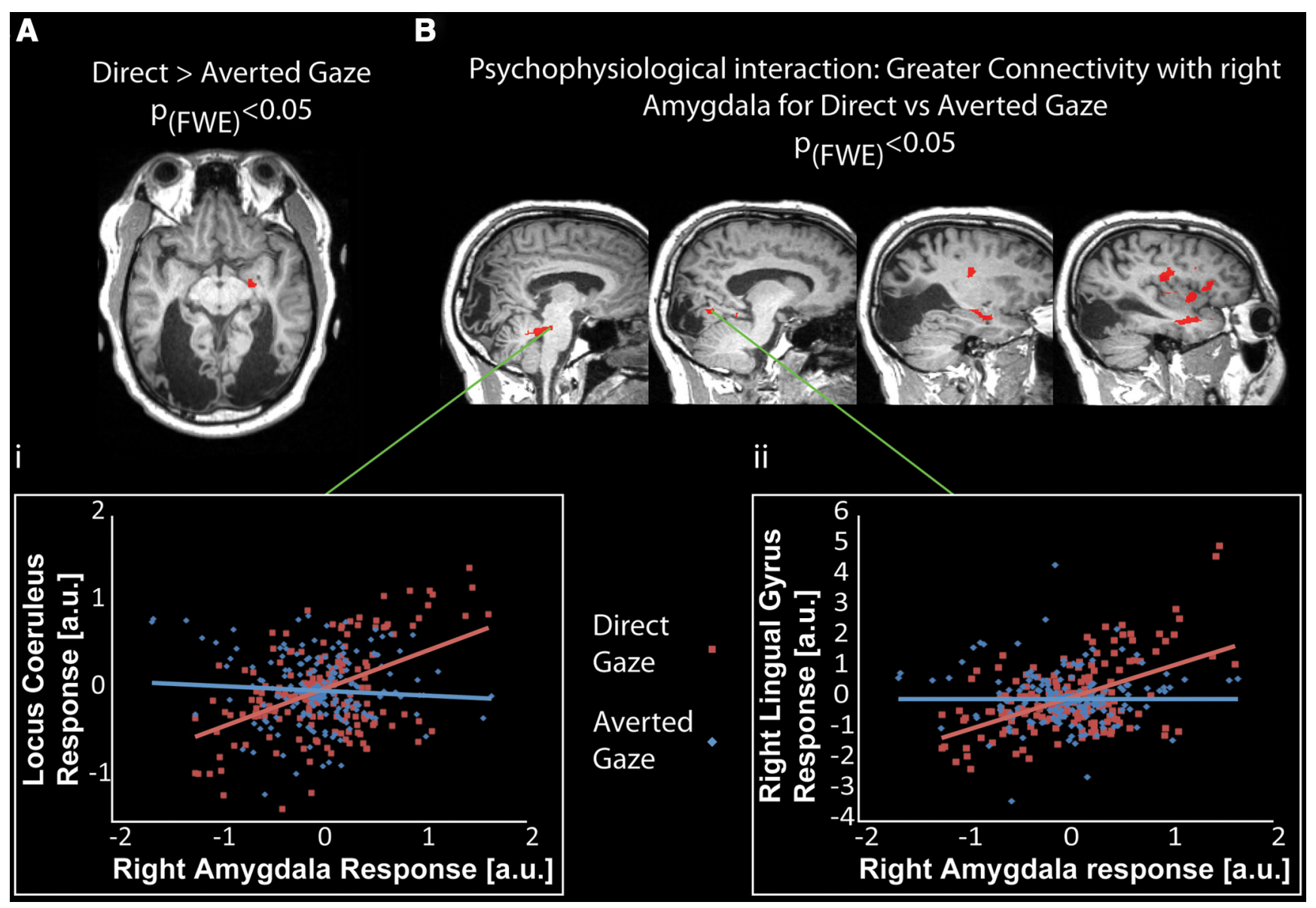

Figure 3. PPI of right amygdala activation with locus ceruleus and right lingual gyrus activation. $\boldsymbol{A}$, Seed region. $\boldsymbol{B}$, Network of regions whose connectivity with the right amygdala was significantly greater in $\mathrm{DG}$ than $\mathrm{AG}\left(p_{\mathrm{fFWE})}<0.05\right.$, cluster extent threshold $\left.k>10\right)$. Scatter plots below show the relative activity, trial by trial, in the amygdala and two regions: (i) locus ceruleus (bottom left), which has previously been shown to respond to subliminal threatening stimuli) (Liddell et al., 2005) and (ii) the right lingual gyrus (bottom right), for DG (red) and AG (blue).

Based on this result, we investigated the functional connectivity of the right amygdala using a PPI technique (Friston et al., 1997). This assessment revealed that, during the presentation of DG versus AG, a significant coactivation of the amygdala was found with several brain areas related to face and gaze processing $\left(p_{\text {(FWE })}<0.05\right)$, including the right lingual gyrus and the right temporal pole, the insula, the hippocampus, and the locus ceruleus (Fig. 3; Table 1).

\section{Control group}

Contrasting activation elicited by DG with that elicited by AG revealed that the right lateral amygdala also exhibits a larger response to DG than AG (Fig. 4; Table 2). In addition, left amygdala and hippocampus as well as the right fusiform gyrus were more activated by gaze contact. No significant main effect of filtering was observed, nor was there a significant interaction between gaze direction and spatial frequency.

\section{ROI analysis: amygdalae}

An ROI analysis was performed using the Marsbar toolbox (Marseille ROI toolbox for SPM8). ROIs were created based on an anatomical mask (WFU_PickAtlas toolbox) (Maldjian et al., 2003 ) for each of the left and the right amygdalae. We then analyzed the data using these masks, which were determined independently of our functional data.

We obtained a clear difference between the DG and AG in the right amygdala for Patient T.N. $\left(t_{(305)}=2.70 p<0.05\right)$ and the control group $\left(t_{(9)}=2.23, p<0.05\right)$. At the single-subject level, the effect DG $>$ AG was significant (at $p<0.05$ ) in two participants and marginally significant in another. In a further five participants, there was a nonsignificant trend for the effect in this direction. In the remaining three participants, the effect was either null $(p \approx 1)$ or showed a nonsignificant trend in the opposite direction.

No difference in activation for DG versus AG was found in the left amygdala for Patient T.N. However, a significant effect of gaze direction was present in the left amygdala for the control group $\left(t_{(9)}=3.43, p<0.01\right)$. At the single-subject level, this effect was significant (at $p<0.05$ ) in three participants, marginally significant in another, and with a nonsignificant trend in five more. The remaining participant showed a nonsignificant trend for the opposite effect.

We also investigated the effect of spatial frequency on amygdala responses to gaze direction. There was no significant main effect of spatial frequency in the amygdalae in either Patient T.N. or the control group. No significant gaze direction by spatial frequency interaction was found either in the left or right amygdala of Patient T.N. or the control group,

\section{Discussion}

Our study shows that, although Patient T.N. lacks a responsive primary visual cortex and has no subjective visual experience of the stimuli presented to him, his right amygdala, in particular the 


\begin{tabular}{|c|c|c|c|c|c|}
\hline \multirow{2}{*}{$\begin{array}{l}\text { Anatomical label } \\
\text { Temporal pole }\end{array}$} & \multirow{2}{*}{$\begin{array}{l}\text { Side } \\
\text { Right }\end{array}$} & \multicolumn{3}{|c|}{ MNI coordinates } & \multirow{2}{*}{$\frac{z \text {-score }}{5.47}$} \\
\hline & & 56 & 4 & -2 & \\
\hline Rolandic operculum & Right & 48; & 0 & 14 & 5.45 \\
\hline Rolandic operculum & Right & 54 & 6 & 2 & 5.4 \\
\hline Hippocampus & Right & 36 & -6 & -22 & 5.27 \\
\hline Brodmann area 13 & Right & 38 & 2 & -24 & 5.25 \\
\hline Insula lobe & Right & 38 & 30 & 6 & 5.2 \\
\hline Insula lobe & Right & $42 ;$ & $2 ;$ & 0 & 5.14 \\
\hline Rolandic operculum & Right & 44 & -12 & 16 & 5.09 \\
\hline Insula lobe & Right & $42 ;$ & 14 & 2 & 5.05 \\
\hline Hippocampus & Right & $32 ;$ & -10 & -18 & 5.01 \\
\hline Insula lobe & Right & $42 ;$ & -14 & 4 & 4.96 \\
\hline Culmen & Left & -10 & -44 & -26 & 5.4 \\
\hline Cerebellum & Left & -12 & -46 & -22 & 5.34 \\
\hline Cerebellum & Left & -12 & -82 & -32 & 5.14 \\
\hline Cerebellum & Right & 8 & -38 & -28 & 5.12 \\
\hline Cerebellum & Right & 6 & $-42 ;$ & -26 & 5.09 \\
\hline Culmen $^{b}$ & Left & -8 & -56 & -24 & 5.03 \\
\hline Periaqueductal gray ${ }^{b}$ & & 0 & -32 & -28 & 4.92 \\
\hline Locus ceruleus $^{b}$ & Left & -4 & -34 & -26 & 4.85 \\
\hline Cerebellum ${ }^{b}$ & Left & -16 & -54 & -26 & 4.83 \\
\hline Cerebellum $^{b}$ & Right & 12 & -46 & -8 & 4.81 \\
\hline Cerebellum ${ }^{b}$ & Left & -24 & -50 & -28 & 4.76 \\
\hline Lingual gyrus $^{b}$ & Right & $14 ;$ & -68 & 2 & 5.18 \\
\hline Lingual gyrus & Right & 14; & -66 & -10 & 4.32 \\
\hline
\end{tabular}

${ }^{a}$ Coordinates of PPI peaks with respect to the comparison DG $>$ AG. Original PPI has been normalized in the MNI space.

${ }^{b}$ Peak voxels of cluster, followed by subpeak voxels a minimum of $8 \mathrm{~mm}$ apart.

lateral aspect, responds more strongly to faces making eye contact than those presenting an AG, using behavioral and brain imaging investigations.

Several studies have emphasized a link between the amygdala, emotions, and gaze processing. Direction of gaze modulates our judgment regarding the intensity of an emotion (Adams and Kleck, 2005); and vice versa, different emotions modify our judgment regarding the direction of gaze (Lobmaier et al., 2008; Lobmaier and Perrett, 2011). These behavioral findings have been corroborated by imaging studies showing that the amygdala response to emotional expressions is modulated by the direction of gaze (Adams and Kleck, 2003; Hadjikhani et al., 2008; Adams et al., 2012). However, in the present study, it is eye contact per se, independently of any emotional expression, that appears to be the critical stimulus. This is not surprising given that gaze is a form of social communication in its own right. For example, investigations have shown that DG produces a greater cortical arousal based on EEG (Gale et al., 1978). Furthermore, DG is associated behaviorally with threat and dominance, inducing avoidance behavior under certain circumstances (reviewed by Kleinke, 1986).

In line with this, imaging studies in healthy controls have found amygdala activity in response to gaze direction with strictly neutral faces as well. In a PET study, Kawashima et al. (1999) compared brain activation in healthy controls who watched videos of a person whose gaze was either averted or directed to the viewer while they judged where the person was looking. When subjects determined gaze direction, the left amygdala was found to be activated relative to a control condition. This was observed whether or not eye contact was made. By contrast, when DG was specifically contrasted with AG, the right amygdala was found to be activated. Subsequently, George et al. (2001) investigated the BOLD response of subjects who viewed photographs of faces with
DG or AG. Compared with AG, DG yielded a stronger activation in ventral occipitotemporal cortices around the fusiform gyrus, activity that was also highly correlated with amygdala activity.

Nevertheless, not all studies have reported amygdala activity when investigating eye contact. Hoffman and Haxby (2000) presented faces with DGs or AGs to their participants, under conditions of passive viewing, or of selective attention to identity or gaze. Overall, their results showed that gaze and identity activated a network of face-processing regions, which comprised the inferior occipital gyrus, lateral fusiform gyrus, and superior temporal sulcus (STS). With regard specifically to gaze processing, we found that passive viewing of AG compared with DG gave rise notably to an increase in left STS activation. STS was interpreted as the structure involved in processing changeable aspect of faces, an explanation that is in line with neurophysiological findings in monkeys (Perrett et al., 1985; Heywood and Cowey, 1992). As the authors point out, the amygdala was not included in their scans; therefore, its contribution cannot be determined. Pageler et al. (2003) performed a similar experiment in which the direction of both the face and gaze were averted or directed to the viewer. The fusiform gyrus and STS both responded to the direction of the face independently of gaze. An effect of eye contact was found but only when faces were also directed toward the viewer. This response was located in the fusiform gyrus, although no amygdala activation was detected.

Consequently, although a certain discrepancy exists in the literature regarding the cerebral regions involved in gaze processing in healthy controls, the results point to a likely involvement of the right amygdala for DG, suggestions that are corroborated by our current investigation. Furthermore, the correlation of amygdala activity with known core face processing areas in our study is in line with another a number of previous reports in the field (Hoffman and Haxby, 2000; George et al., 2001; Pageler et al., 2003).

First and foremost in our study, however, is the finding that amygdala activation occurs regardless of the loss of V1. One plausible explanation for this stems from a suggestion put forward by LeDoux (1996) that a coarse but rapid subcortical pathway, bypassing V1, might convey information about potentially threatening stimuli to the amygdala, allowing appropriate response behavior to be implemented more quickly. In case of damage to the primary sensory cortices, this route would allow behaviorally relevant stimuli to be processed without awareness. Strikingly, the existence of a response for DG in our study demonstrates that eye contact belongs to this general category of relevant stimuli along with emotional facial expressions and that blindsight for gaze can occur.

In Patient T.N., affective blindsight was previously observed, and data had revealed that emotional faces activated his right amygdala despite his cortical blindness (Pegna et al., 2005), which seemed to support the existence of such a subcortical route. Evidence from another blindsight patient was obtained by Morris et al. (2001) showing amygdala activation for unseen faces that was correlated with activity in the superior colliculus and pulvinar, thereby supporting the existence of a colliculo-pulvino-amygdalar pathway in blindsight.

Lately, the existence of this subcortical route has been the subject of considerable debate, and other pathways (e.g., thalamoextrastriate routes) have been put forward as possible alternatives) (Pessoa and Adolphs, 2010; Tamietto and de Gelder, 2010). More recently, however, a diffusion tensor imaging study was performed on another, unilateral blindsight patient (G.Y.) as well as a group of controls. Neural connections between the superior 
colliculus, pulvinar, and amygdala were found in both the control group and the patient. The modifications observed in the patient suggested that this pathway could indeed constitute the basis for the residual function observed in affective blindsight (Tamietto et al., 2012). Although our current study does not allow a precise pathway to be established, it nevertheless demonstrates unequivocally that certain categories of stimuli do not necessitate the primary visual cortex to access the amygdala. Eye contact clearly belongs to this category.

Along the lines suggested by LeDoux (2012), if eye contact represents a possible threat, it would thus constitute a key stimulus that could be processed automatically to trigger the appropriate responses. As noted by LeDoux (2012), the circuits underlying defense responses triggered by unconditioned threat stimuli would include the lateral amygdala, as well as the periaqueductal gray that constitutes part of the defensive response, both of which were found to be active in our study.

The amygdala (and periaqueductal gray) that we observe could therefore be part of an automatic defense circuit operating without awareness and without detailed processing by the primary visual cortex. Indeed, the amygdala can be triggered by relatively crude stimuli. For example, Vuilleumier et al. (2003) showed that the low spatial frequency components of a fearful face were sufficient to trigger an amygdala response. However, our results showed that spatial frequency had no effect on the fMRI response (the effect of gaze direction on amygdala activation was equally strong in all three filtering conditions). Thus, in our study, the relevant visual cues did not appear to depend strictly on the low spatial frequency components, which appears to argue against the view that the amygdala relies solely on coarse, low spatial frequency information. However, Whalen et al. (2004) observed that the whites of eyes expressing fear produced a greater response in the amygdala compared with eye whites of happy expressions. This was observed even though the stimuli were masked and the participants unaware of their presence. Building on this hypothesis, we would surmise that, if the crude information conveyed by the whites of the eyes is sufficient to trigger a response in the amygdala indicating fear, this explanation should be even more compelling pertaining to gaze direction. Gaze processing is possible through the specific pattern formed by the sclera that is modified depending on the direction of orientation of the eyes. Because the information contained in the whites of the eye is sufficient to drive an amygdala response, it is likely that this structure can also be triggered by DG (Kobayashi and Kohshima, 1997) and may nonetheless arise through a subcortical pathway even for high spatial frequency stimuli. Future studies should attempt to address the issue of spatial frequency in more depth.

In conclusion, this investigation shows that eye contact is processed by the amygdala in this patient, through pathways that must necessarily bypass the primary visual cortex. Because direct gaze possesses an important behavioral value in terms of danger or submission and of social interaction in general among conspecifics, it is likely to constitute a powerful signal that can activate a
Table 2. Significant differences in BOLD response for direct versus averted gaze (uncorrected $p<0.001$, with a cluster extent threshold of 10 voxels)

\begin{tabular}{|c|c|c|c|}
\hline Anatomical label & Side & MNI coordinates & $z$-score \\
\hline Hippocampus $^{a}$ & Left & $-22 ;-16 ;-18$ & 3.69 \\
\hline Amygdala & Left & $-32 ;-6 ;-20$ & 3.67 \\
\hline Parahippocampal gyrus & Left & $-30 ;-28 ;-16$ & 3.4 \\
\hline Thalamus $^{a}$ & Right & $8 ;-6 ; 0$ & 4.23 \\
\hline Middle occipital gyrus ${ }^{a}$ & Left & $-28 ;-84 ; 4$ & 4.19 \\
\hline Fusiform gyrus $^{a}$ & Right & $34 ;-48 ;-12$ & 4.15 \\
\hline Posterior insula ${ }^{a}$ & Right & $46 ; 2 ;-8$ & 3.79 \\
\hline White matter (anterior commissure) ${ }^{a}$ & Right & $6 ; 6 ; 6$ & 3.73 \\
\hline Hypothalamus $^{a}$ & Right & $6 ;-2 ;-16$ & 3.52 \\
\hline Parahippocampal gyrus & Right & $16 ;-2 ;-20$ & 3.2 \\
\hline Hippocampus $^{a}$ & Right & $24 ;-14 ;-20$ & 3.46 \\
\hline Lingual gyrus $^{a}$ & Right & $24 ;-86 ;-6$ & 3.42 \\
\hline
\end{tabular}

${ }^{a}$ Peak voxels of cluster, followed by subpeak voxels a minimum of $8 \mathrm{~mm}$ apart.

circuit underlying defense responses without information from $\mathrm{V} 1$ and thus without awareness.

\section{References}

Adams RB Jr, Kleck RE (2003) Perceived gaze direction and the processing of facial displays of emotion. Psychol Sci 14:644-647. CrossRef Medline

Adams RB Jr, Kleck RE (2005) Effects of direct and averted gaze on the perception of facially communicated emotion. Emotion 5:3-11. CrossRef Medline

Adams RB Jr, Franklin RG Jr, Kveraga K, Ambady N, Kleck RE, Whalen PJ, Hadjikhani N,Nelson AJ (2012) Amygdala responses to averted vs direct gaze fear vary as a function of presentation speed. Soc Cogn Affect Neurosci 7:568-577. CrossRef Medline

Ashburner J, Friston KJ (2005) Unified segmentation. Neuroimage 26:839_ 851. CrossRef Medline

Crinion J, Ashburner J, Leff A, Brett M, Price C, Friston K (2007) Spatial normalization of lesioned brains: performance evaluation and impact on fMRI analyses. Neuroimage 37:866-875. CrossRef Medline

de Gelder B, Vroomen J, Pourtois G, Weiskrantz L (1999) Non-conscious recognition of affect in the absence of striate cortex. Neuroreport 10: 3759-3763. CrossRef Medline

Delplanque S, N'diaye K, Scherer K, Grandjean D (2007) Spatial frequencies or emotional effects? A systematic measure of spatial frequencies for IAPS pictures by a discrete wavelet analysis. J Neurosci Methods 165:144150. CrossRef Medline

Eickhoff SB, Stephan KE, Mohlberg H, Grefkes C, Fink GR, Amunts K, Zilles K (2005) A new SPM toolbox for combining probabilistic cytoarchitec- 
tonic maps and functional imaging data. Neuroimage 25:1325-1335. CrossRef Medline

Ethofer T, Gschwind M, Vuilleumier P (2011) Processing social aspects of human gaze: a combined fMRI-DTI study. Neuroimage 55:411-419. CrossRef Medline

Friston KJ, Buechel C, Fink GR, Morris J, Rolls E, Dolan RJ (1997) Psychophysiological and modulatory interactions in neuroimaging. Neuroimage 6:218-229. CrossRef Medline

Gale A, Kingsley E, Brookes S, Smith D (1978) Cortical arousal and social intimacy in the human female under different conditions of eye contact. Behav Proc 3:271-275. CrossRef

George N, Driver J, Dolan RJ (2001) Seen gaze-direction modulates fusiform activity and its coupling with other brain areas during face processing. Neuroimage 13:1102-1112. CrossRef Medline

Hadjikhani N, Hoge R, Snyder J, de Gelder B (2008) Pointing with the eyes: the role of gaze in communicating danger. Brain Cogn 68:1-8. CrossRef Medline

Heywood CA, Cowey A (1992) The role of the 'face-cell' area in the discrimination and recognition of faces by monkeys. Philos Trans $\mathrm{R}$ Soc London 335:31-37; discussion 37-38. CrossRef Medline

Hoffman EA, Haxby JV (2000) Distinct representations of eye gaze and identity in the distributed human neural system for face perception. Nat Neurosci 3:80-84. CrossRef Medline

Holmes G (1918) Disturbances of vision by cerebral lesions. Br J Ophthalmol 2:353-384. CrossRef Medline

Kawashima R, Sugiura M, Kato T, Nakamura A, Hatano K, Ito K, Fukuda H, Kojima S, Nakamura K (1999) The human amygdala plays an important role in gaze monitoring: a PET study. Brain 122:779-783. CrossRef Medline

Kleinke CL (1986) Gaze and eye contact: a research review. Psychol Bull 100:78-100. CrossRef Medline

Kobayashi H, Kohshima S (1997) Unique morphology of the human eye. Nature 387:767-768. CrossRef Medline

LeDoux J (1996) Emotional networks and motor control: a fearful view. Prog Brain Res 107:437-446. CrossRef Medline

LeDoux J (1998) Fear and the brain: where have we been, and where are we going? Biol Psychiatry 44:1229-1238. CrossRef Medline

LeDoux J (2012) Rethinking the emotional brain. Neuron 73:653-676. CrossRef Medline

Liddell BJ, Brown KJ, Kemp AH, Barton MJ, Das P, Peduto A, Gordon E, Williams LM (2005) A direct brainstem-amygdala-cortical 'alarm' sys- tem for subliminal signals of fear. Neuroimage 24:235-243. CrossRef Medline

Lobmaier JS, Tiddeman BP, Perrett DI (2008) Emotional expression modulates perceived gaze direction. Emotion 8:573-577. CrossRef Medline

Lobmaier JS, Perrett DI (2011) The world smiles at me: self-referential positivity bias when interpreting direction of attention. Cogn Emot 25:334-341. CrossRef Medline

Maldjian JA, Laurienti PJ, Kraft RA, Burdette JH (2003) An automated method for neuroanatomic and cytoarchitectonic atlas-based interrogation of fMRI data sets. Neuroimage 19:1233-1239. CrossRef Medline

Morris JS, DeGelder B, Weiskrantz L, Dolan RJ (2001) Differential extrageniculostriate and amygdala responses to presentation of emotional faces in a cortically blind field. Brain 124:1241-1252. CrossRef Medline

Pageler NM, Menon V, Merin NM, Eliez S, Brown WE, Reiss AL (2003) Effect of head orientation on gaze processing in fusiform gyrus and superior temporal sulcus. Neuroimage 20:318-329. CrossRef Medline

Pegna AJ, Khateb A, Lazeyras F, Seghier ML (2005) Discriminating emotional faces without primary visual cortices involves the right amygdala. Nat Neurosci 8:24-25. CrossRef Medline

Perrett DI, Smith PA, Potter DD, Mistlin AJ, Head AS, Milner AD, Jeeves MA (1985) Visual cells in the temporal cortex sensitive to face view and gaze direction. Proc R Soc Lond B Biol Sci 223:293-317. CrossRef Medline

Pessoa L, Adolphs R (2010) Emotion processing and the amygdala: from a 'low road' to 'many roads' of evaluating biological significance. Nat Rev 11:773-783. CrossRef Medline

Tamietto M, de Gelder B (2010) Neural bases of the non-conscious perception of emotional signals. Nat Rev 11:697-709. CrossRef Medline

Tamietto M, Pullens P, de Gelder B, Weiskrantz L, Goebel R (2012) Subcortical connections to human amygdala and changes following destruction of the visual cortex. Curr Biol 22:1449-1455. CrossRef Medline

Vuilleumier P, Armony JL, Driver J, Dolan RJ (2003) Distinct spatial frequency sensitivities for processing faces and emotional expressions. Nat Neurosci 6:624-631. CrossRef Medline

Weiskrantz L (1986) Some aspects of memory functions and the temporal lobes. Acta Neurol Scand 109:69-74. Medline

Weiskrantz L, Warrington EK, Sanders MD, Marshall J (1974) Visual capacity in the hemianopic field following a restricted occipital ablation. Brain 97:709-728. CrossRef Medline

Whalen PJ, Kagan J, Cook RG, Davis FC, Kim H, Polis S, McLaren DG, Somerville LH, McLean AA, Maxwell JS,Johnstone T (2004) Human amygdala responsivity to masked fearful eye whites. Science 306:2061. CrossRef Medline 